\title{
Computers and Advanced Instrumentation
}

National Cancer Institute

\section{Source}

National Cancer Institute. Computers and Advanced Instrumentation. NCI Thesaurus.

Code 15884.

NIH Emphasis Area -- Recent advances in basic biology, genetics, diagnosis, and health care have all been closely linked to the development of new research instruments and computer hardware and software. Improvements in the detection of early cancers and technologies to better visualize the living body in health and disease states will provide a wealth of knowledge that can lead to new and improved diagnostics, treatments and prevention strategies. 